\title{
SOLAR ACTIVITY OBSERVED WITH THE NEW NOBEYAMA RADIOHELIOGRAPH
}

\author{
Shinzo Enome, Hiroshi Nakajima, Kiyoto Shibasaki, \\ Masanori Nishio, Toshiaki Takano, Yoichiro Hanaoka, \\ Chikayoshi Torii, Hideaki Sekiguchi, Susumu Kawashima, \\ Takeshi Bushimata, Noriyuki Shinohara, Yoshihisa Irimajiri, \\ Hideki Koshiishi, Takeo Kosugi, Yasuhiko Shiomi, Masaki Sawa, \\ and Keizo Kai*
}

Nobeyama Radio Observatory

Minamisaku, Nagano, 384-13 Japan

* passed away in March, 1991

\begin{abstract}
The new Nobeyama Radioheliograph was completed in March 1992 after two years of construction. It is a T-shaped array operating as a multiple spacing grating-type radio interferometer at 17 $\mathrm{GHz}$ and is dedicated to full disk solar observations. Routine observations began in late June, 1992, after three months of system integration, fine tuning, and test observations. During the course of test observations it was shown that major items of the system performance exceeded the designed values, and that the image quality or the dynamic range of the images is better than the designed value. In the three months of routine observations two X-class flares, several M-class flares and a number of small flares were observed. In this report we present a summary of initial observational results and preliminary comparisons with YOHKOH HXT and SXT observations.
\end{abstract}

\section{ANTENNA SITE}

The array was constructed at Nobeyama Radio Observatory, which is in the Nagano prefecture, located about $160 \mathrm{~km}$ west of Mitaka, Tokyo, and is a two- or three-hour drive from Mitaka. The radioheliograph is the third major instrument after the 45-m diameter dish and the 5-element $10-\mathrm{m}$ diameter $\mathrm{mm}$-wave interferometer which were constructed about ten and five years ago respectively for cosmic radio observations. A general view of the antennas is shown in Figure 1. 
Construction of the Radioheliograph started in April, 1990 at Nobeyama. The array design emphasizes quick and easy calibration using the unique extended radio source, namely, the Sun itself. This is compatible with observational requirements such as a wide field of view (full disk of the Sun), and high image quality (better than $20 \mathrm{~dB}$ ) in the snapshot mode. Element antennas are 80$\mathrm{cm}$ diameter paraboloids to cover the full solar disk at $17 \mathrm{GHz}$. The antenna configuration is a multiple-spacing grating-type $\mathrm{T}$-shaped array tapered to give higher weight at shorter spacings which enables us to directly apply the fast Fourier transform algorithm in two dimensions and to make accurate determinations of the quiet Sun or solar disk. In order to guarantee high stability of the complex gain, low-loss phase-stable optical fiber cables $(<0.2$ $\mathrm{ppm} /$ degree) are extensively used as transmission lines. One bit correlators are employed with custom CMOS $4 \times 4$ gate arrays and LSI circuits with a 10 $\mathrm{MHz}$ clock in four-phase operation to realize a $40 \mathrm{MHz}$ sampling rate. A highspeed computer of 285 MFLOPS is employed to obtain real-time images of the Sun at 10 -sec intervals, which are recorded on a video tape simultaneously and used later for quick-look data. The 3486 combinations of correlator outputs are recorded at $50 \mathrm{msec}$ on a commercial digital video recorder, and 1-sec integrated data are recorded on computer storage, which are also synthesized into images using the high-speed computer. Image restoration software was developed by our group. An important new factor which is applied in the software algorithm, is that we have to precisely estimate the quiet solar disk, which is then convolved with the dirty beam and subtracted from the observed dirty map. The dynamic range thus obtained is better than $20 \mathrm{~dB}$. A more detailed description of the Nobeyama Radioheliograph system is given by Nakajima et al (1993).

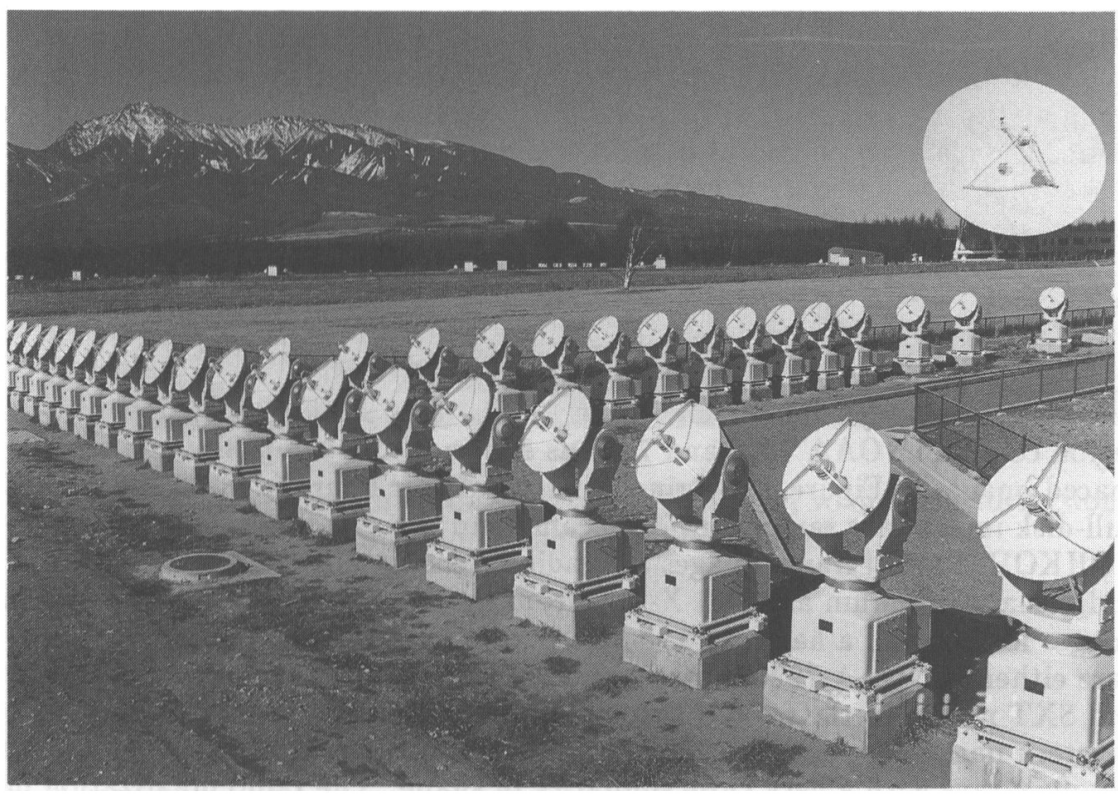

Fig. 1. General view of the Nobeyama Radioheliograph. 


\section{EXAMPLES OF INITIAL MORPHOLOGY RESULTS}

Radio emission from the Sun at short centimeter wavelengths arises from both thermal and non-thermal sources, which are shown in the following examples of initial observations. A very brief survey of observed events indicates that thermal free-free emission, or Bremsstrahlung, is not strongly polarized, whereas gyroresonance thermal radiation and impulsive non-thermal radiation are strongly polarized. Comparisons with $\mathrm{YOHKOH}$ observations reveal that there is a close similarity between soft X-ray emission and thermal free-free radiation in shape and evolution. Gyroresonance and non-thermal radiations, on the other hand, are not seen in images from the soft X-ray telescope (SXT) of $\mathrm{YOHKOH}$.

\section{A. Disk Component}

Thermal radiation from the solar disk is illustrated in Figure 2 (1992 July 7), in which a 9 percent sensitivity gradient across the field of each of the array's antennas has been corrected. The map shows limb brightening of 7 percent due to the coronal contribution at $17 \mathrm{GHz}$. The estimated quiet Sun disk is larger than the optical disk by $1.25 \%$ or $9000 \mathrm{~km}$, which is considered to correspond to the chromospheric contribution. Accumulation of these data for a long period of time will give us the structure of the solar atmosphere from the chromosphere to the corona. Note an eruptive prominence above the west limb.

\section{B. Eruptive Prominence and An Arcade Formation}

A spectacular scene of an eruptive prominence on 1992 July 31 is presented in Figure 3. This is a superposition of three time-spaced radio images above the disk and an SXT image of the disk in negative form. The formation of an arcade below the eruptive prominence and just inside the north-west limb is seen as a reconnection of the magnetic field. This event was also observed in $\mathrm{H}$ alpha at Hida, Norikura, and Mitaka. The total duration of the event is several hours. This is a best example of a classic disparition brusque using advanced observational techniques. It is also the observational manifestation of flare models proposed by Carmichel(1964), Sturrock(1968), Hirayama(1974), and Kopp and Pneuman(1976). Morphological and quantitative analyses are in progress.

\section{Micro Activity or Rapid Evolution of Active Regions}

An example of very small or micro activity or rapid evolution is the active region complex NOAA 7220 and 7222 as shown in the composite and timespaced images of Figure 4 covering a period of four hours from 0050-0450 UT. Full-disk images are radio from Nobeyama and the visual continuum from YOHKOH. Partial frame images are radio intensity and radio polarization, and visual continuum and soft X-rays from the YOHKOH SXT. The first four images for one and a half hours show the remnants or decaying phase of a flare either in NOAA 7220 or between the two sunspot groups in both radio and SXT maps. In the 0250 UT maps, new emission is seen in NOAA 7222 (western part of the active region) in radio intensity and polarization, and also in soft X-rays with a very close similarity in shape. The radio polarization map 


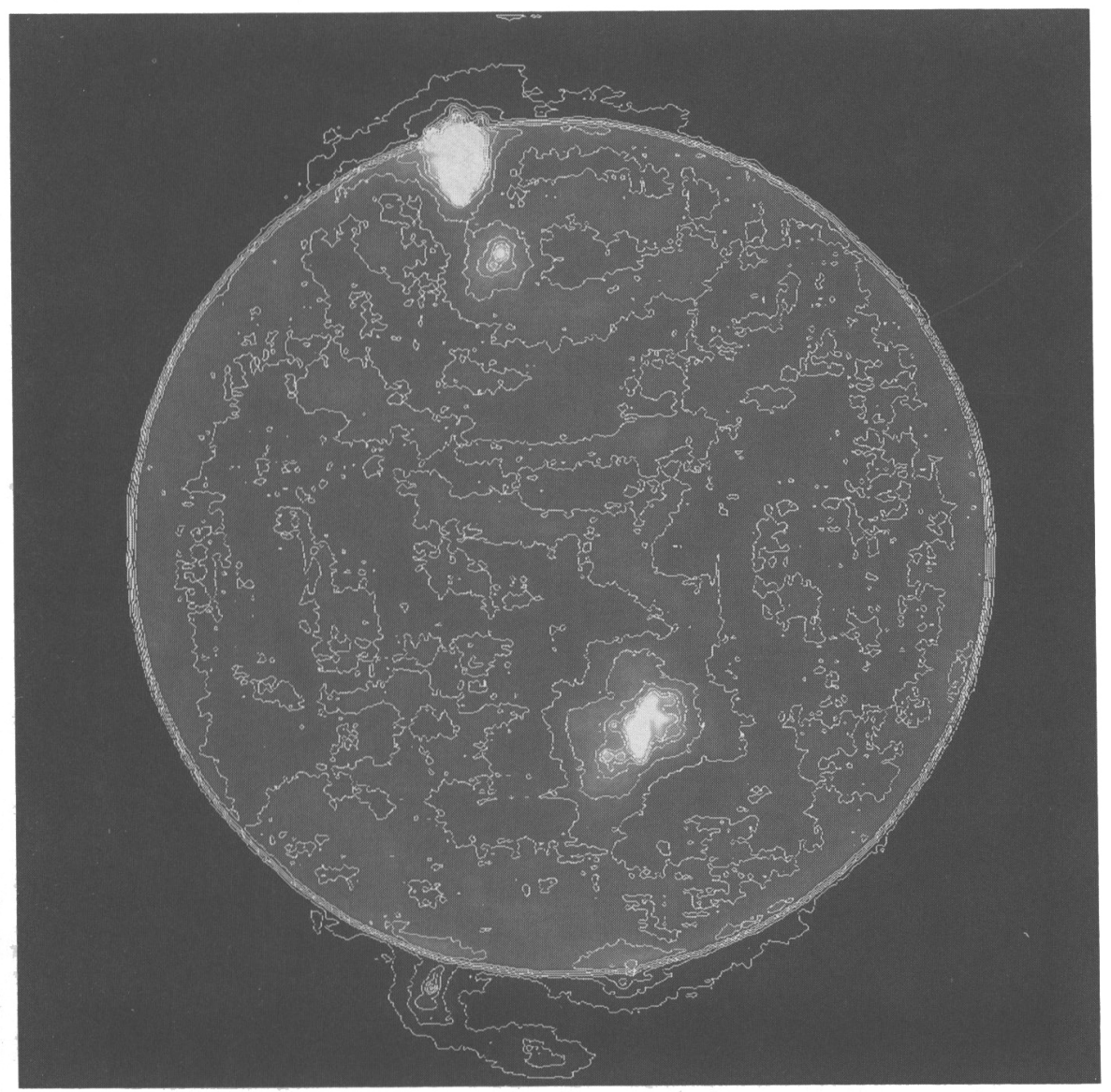

Fig. 2. A radio map on 1992 July 7 is shown with a $9 \%$ correction for non-uniformity in element antenna illumination. 
S. ENOME ET AL.

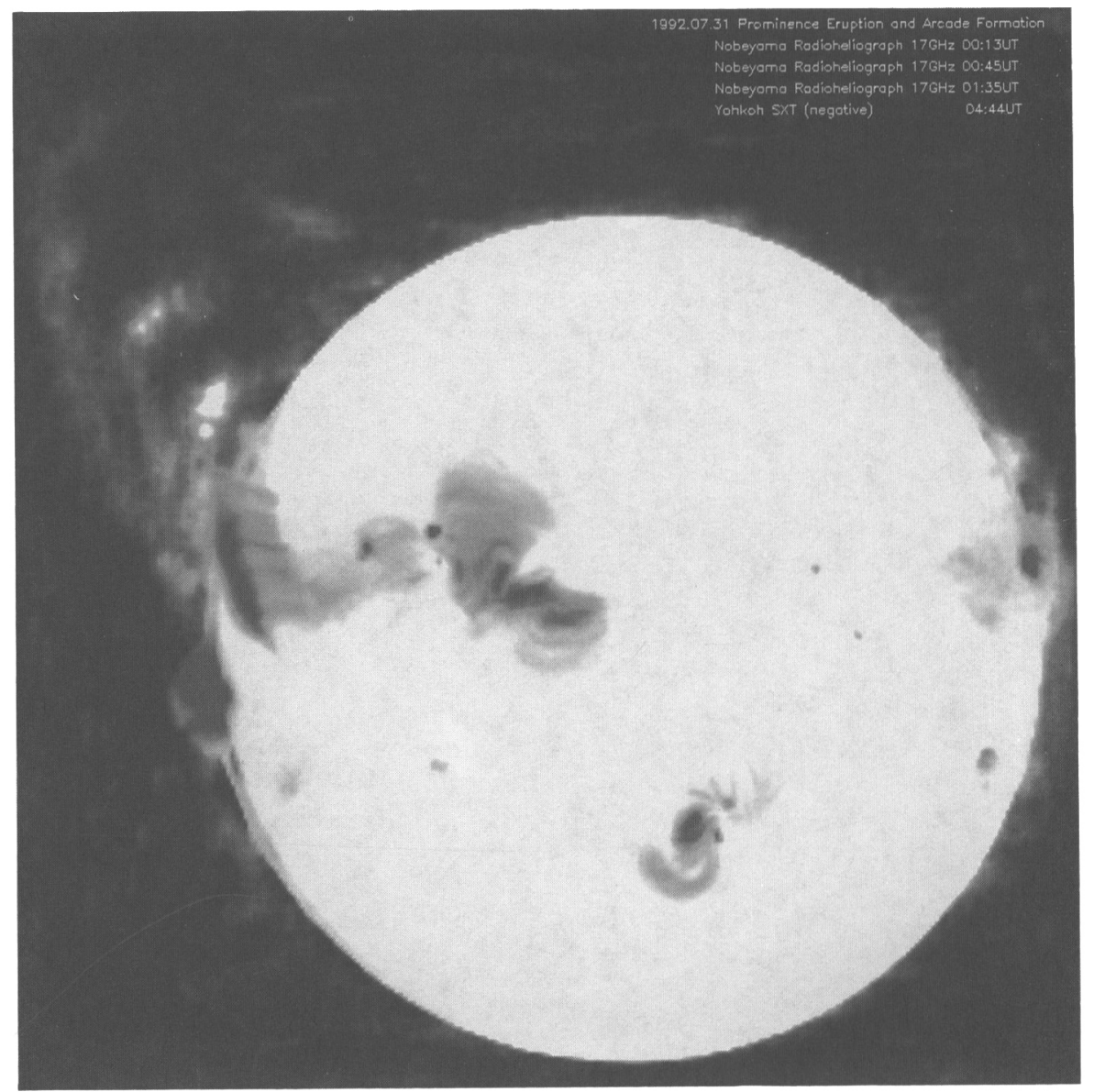

Fig. 3. An eruptive prominence and formation of an arcade on 1992 July 31 . This is a composite of three radio images above the solar disk, covering one and a half hours, and a negative disk image from the YOHKOH SXT at a later phase of the arcade formation. 


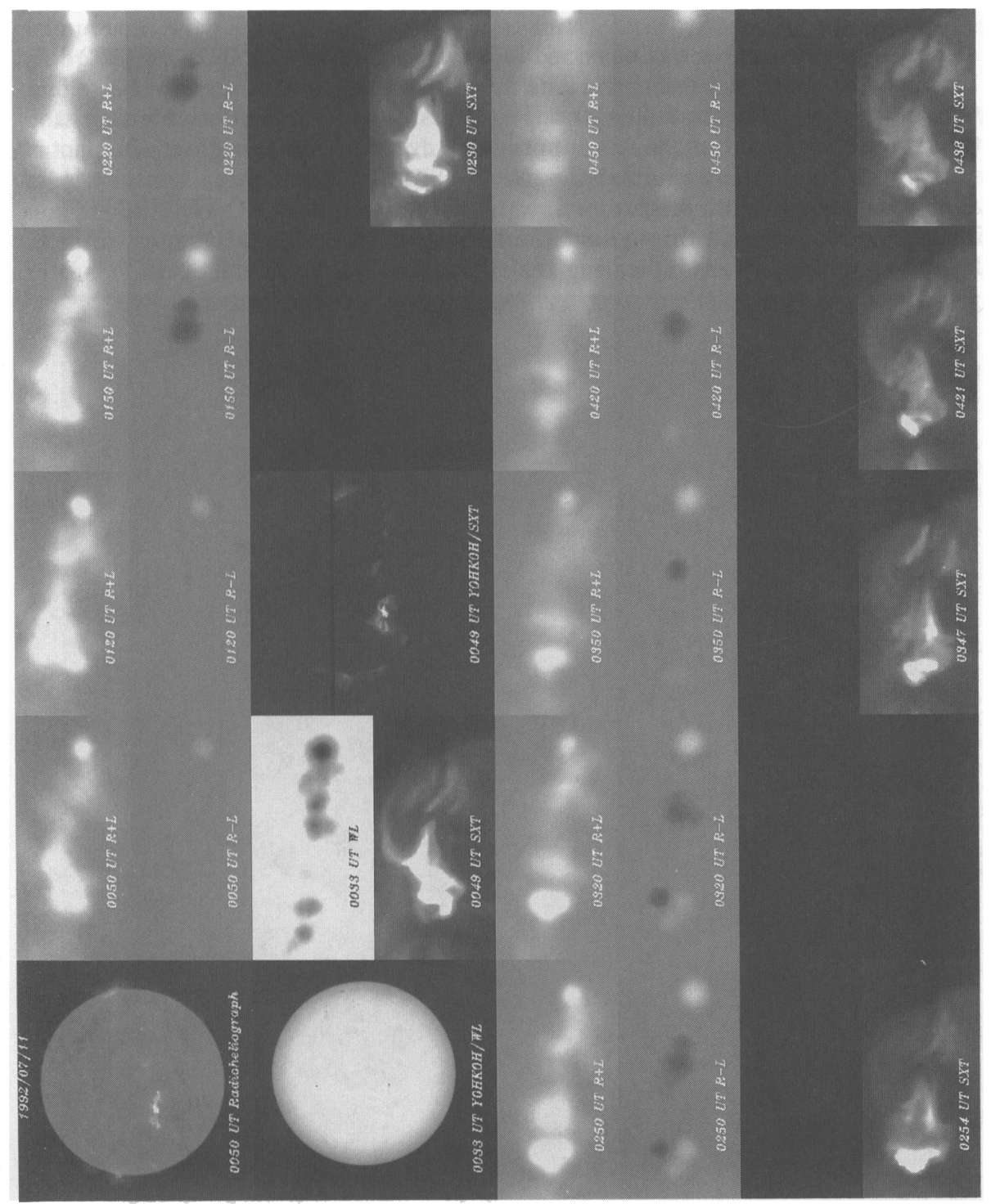

Fig. 4. Micro-activity during four hours on 1992 July 11 observed in a complex active region NOAA $7220 / 7222$ with the Nobeyama Radioheliograph, YOHKOH SXT white light and soft X-rays. 
shows three polarized components in NOAA 7222 , which prove to be consistent with the extraordinary mode of propagation.

\section{Loop-Loop Interaction}

A loop-loop interaction was observed in active region NOAA 7226 on $1992 \mathrm{July}$ 15. Figure 5 shows its evolution. The first subframe shows a preflare stage of two weak loops. The second subframe demonstrates an initial brightening at the intersection point of the two loops which evolved into a brighter compact source in several minutes. After the maximum phase, the compact source faded as if it redistributed its brightness into the two loops. The YOHKOH SXT images for preflare and throughout the flare show quite similar shapes to the Radioheliograph images indicating the thermal character of the event.

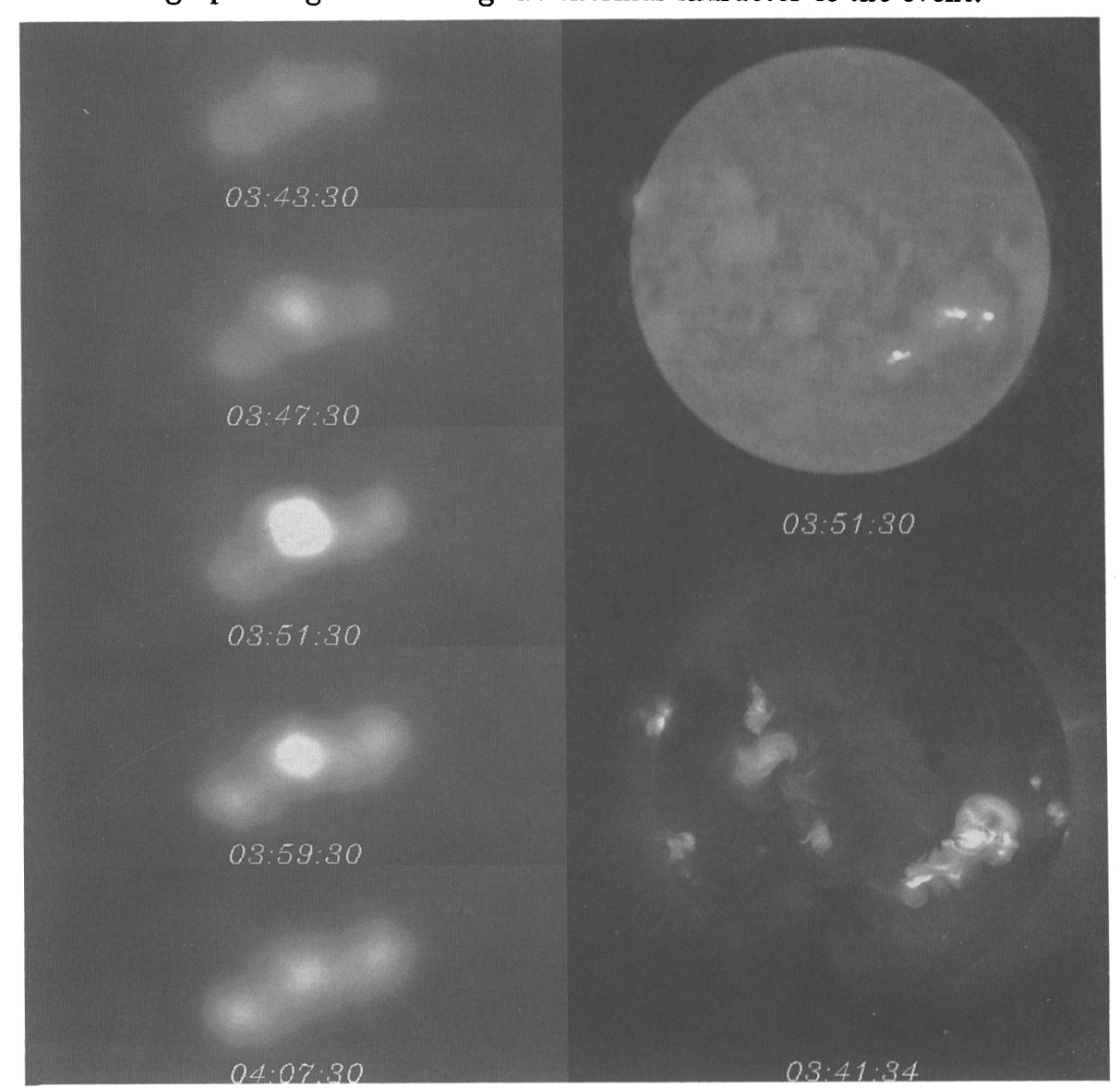

Fig. 5. Loop-loop interaction observed in NOAA 7226 on 1992 July 15. An interaction took place at the center of each subframe, where brightening started and subsided in the decay phase with gradual brightening of entire loops. 


\section{E. An Example of Possible Non-Thermal and Thermal Causality}

An M-class flare (according to GOES measurements) was observed at the east limb of the Sun on 1992 June 28. The flare was composed of a gradual thermal component with a duration of two hours, and occasional non-thermal impulsive components. Figure 6 shows the spatial structures of both the thermal and non-thermal components. At $0228 \mathrm{UT}$, a brightening of an impulsive component appeared as a compact, strongly polarized source. It then gradually changed its shape into an upward, elongated thermal component. One hour and twenty minutes later (0404 UT) another brightening of the original non-thermal point appeared along with two bright thermal points at higher altitudes. The latter thermal components are seen with good shape agreement in the YOHKOH SXT image, while the former non-thermal component, which later evolved into a thermal component, is not seen in YOHKOH images.

\section{F. Impulsive Event of 1992 August 17}

In active region NOAA 7260 an impulsive event occurred in association with an emerging flux region. The radio source showed a double structure in the initial and maximum phases as illustrated in Figure 7. After the maximum phase, the source showed less conspicuous substructure and evolved into an elongated structure in the declining phase. This structural evolution suggests that radio emission in the impulsive and the maximum phases originates at the foot-points of a loop, while in the declining phase radio emission is confined to the loop-top. This is, however, not compatible with the observed polarization structure, since the polarization maps in Figure 7 do not exhibit bipolar components but rather double components both lefthand circularly polarized. There are several other impulsive events showing similar polarization structure. The polarization sense of active regions and gradual bursts is in the extraordinary mode, which agrees with theory. Several attempts are in progress to resolve this new puzzle.

\section{A PLAN OE DATA ANALYSES}

Analyses of the first results of observations by host scientists, including most of the items briefly described above, are in progress, and will be finalized as initial papers in early 1993. A limited number of joint analyses of events observed in common with YOHKOH are also in progress by host and guest scientists as provisional open use of the Nobeyama Radioheliograph data. Full-scale open use of data is scheduled in 1993. Allocation of events to each individual guest scientist is prepared by a program committee, which should be organized soon. Release of the Nobeyama Radioheliograph data in the form of the observation time log, event list, and a limited number of maps is also under way. The expected schedule is early 1993 .

\section{SCIENCE MEETING}

A science symposium featuring $\mathrm{YOHKOH}$ and Radioheliograph data analyses will be held on 6-10 September 1993 in Kofu, Yamanashi prefecture. The title of the meeting is "New Look at the Sun, Dynamic Solar Corona and Flares", with a sub-title "What do we see with YOHKOH and Nobeyama 


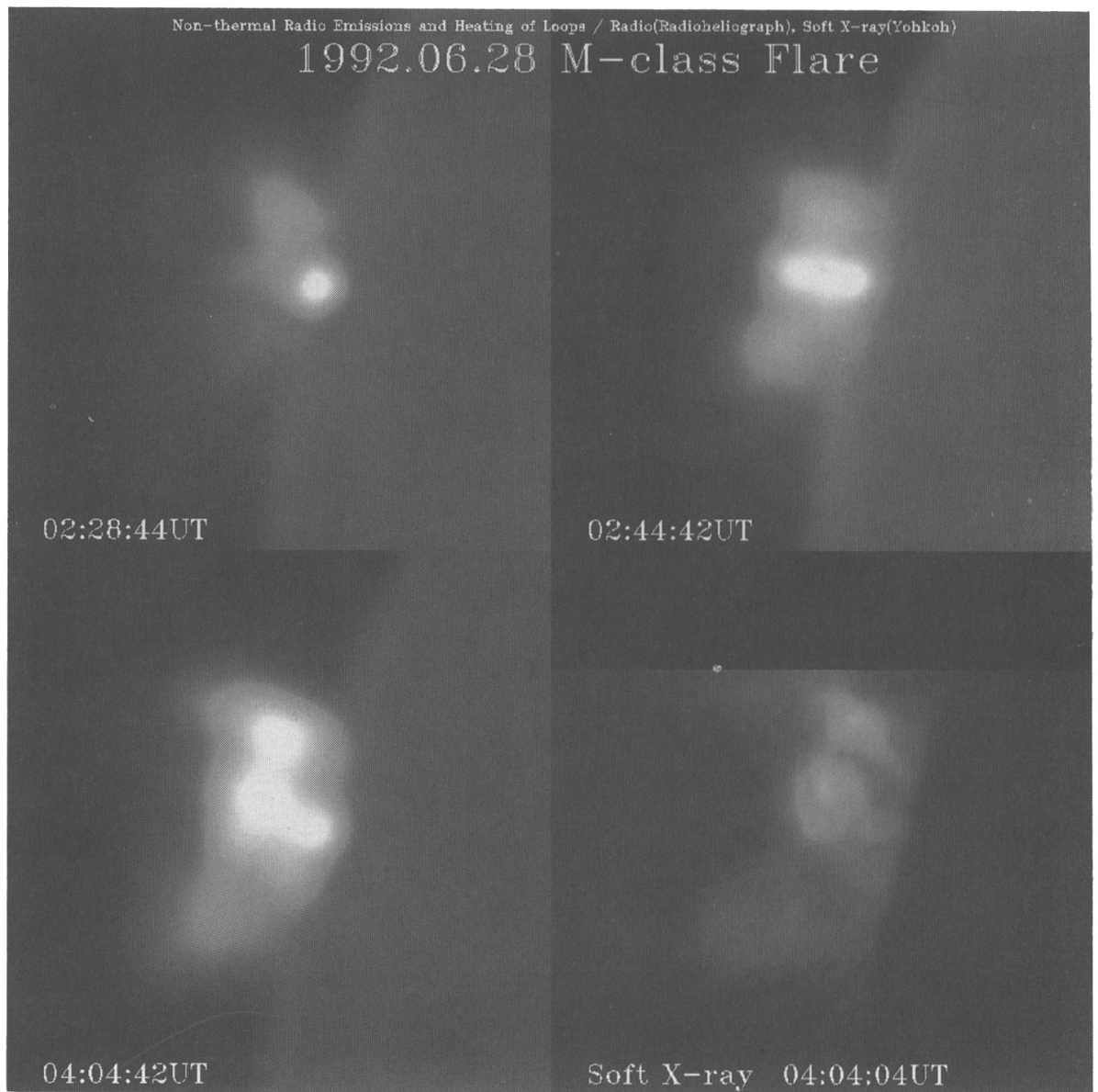

Fig. 6. A thermal and non-thermal relation is illustrated in the M-class flare on 1992 June 28 at the east limb of the Sun. A time-impulsive component shows as a strongly polarized, compact source in the first subframe, and evolves into an elongated thermal source in the second subframe. In the third subframe, two thermal sources at higher altitudes and a new non- thermal source at the original position revived. The thermal sources are similar in shape and position with the SXT image in the fourth subframe while the non-thermal source is not seen. 


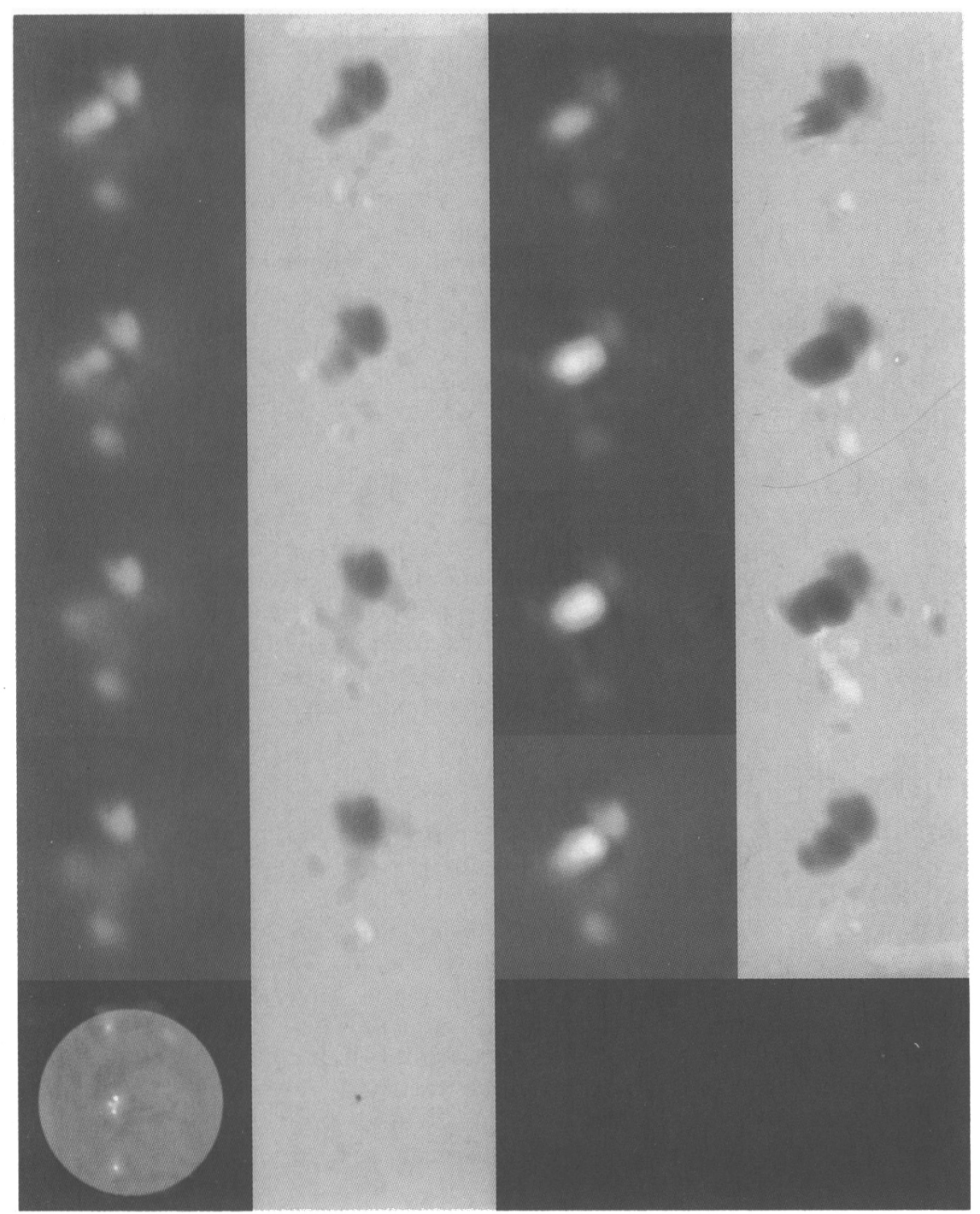

Fig. 7. Intensity and polarization of an impulsive event on 1992 August 18. During the impulsive and maximum phases the source is composed of double components in intensity but is not bipolar in polarization. 
Radioheliograph". The first circular was distributed in December, 1992.

Second circular will be distributed in March, 1993 and submission of abstracts is scheduled for June, 1993.

\section{FUTURE UPGRADE}

Upgrades of the Nobeyama Radioheliograph are considered in two ways. The first is dual frequency observations with a new frequency at $34 \mathrm{GHz}$, which will give us radio spectra of flares in the optically thin domain. The second is an increased number of element antennas which will give us better image quality and enable us to observe low contrast structures in the chromosphere.

\section{SUMMARY AND CONCLUSION}

In this report we have shown some of the potential of the Nobeyama Radioheliograph revealed in the initial stages of observations. This facility promises to increase our knowledge about high energy processes associated with solar flare phenomena. Schedules are given for possible collaborative studies with the Nobeyama Radioheliograph data and also for a science meeting featuring the Radioheliograph data and YOHKOH data analyses. Finally, a concept for upgrading of the Radioheliograph is presented.

\section{REFERENCES}

Carmichael, H., 1964, AAS-NASA Symposium on the Physics of Solar Flares, NASA-SP 50, W. N. Hess (ed.), 451.

Hirayama, T., 1974, Solar Phys., 34, 323.

Kopp, R. A. \& Pneuman, G. W., 1976, Solar Phys., 50, 85.

Nakajima, H., Enome, S., Shibasaki, K., Nishio, M., Takano, T., Hanaoka, Y., Torii, C., Sekiguchi, H., Bushimata, T., Kawashima, S., Shinohara, N., Irimajiri, Y., Koshiishi, H., Kosugi, T., Shiomi, Y., Sawa, S., \& Kai, K., 1993, "The Nobeyama Radioheliograph", Proc. IEEE, Special Issue Design and Instrumentation of Antennas for Deep Space Telecommunications and Radio Astronomy, in press.

Sturrock, P. A., 1968, IAU Symposium No. 35, 471. 